\title{
User Assignment for MIMO-OFDM Systems with Multiuser Linear Precoding
}

\author{
Hassen Karaa and Raviraj S. Adve \\ Dept. of Electrical and Computer Engineering, University of Toronto \\ 10 King's College Road, Toronto, Ontario, M5S 3G4, Canada \\ Email: \{hkaraa,rsadve\}@comm.utoronto.ca
}

\begin{abstract}
Recent work has developed single-carrier linear precoding for the multiuser downlink for multiple-input multipleoutput (MIMO) systems. This paper addresses the problem of user selection in orthogonal frequency division multiplexing (OFDM) based MIMO systems using such multiuser precoding. We propose an effective scheme to assign multiple users, chosen from a larger set, to individual subcarriers. The scheme is developed in the context of a previously proposed single-carrier MIMO linear precoding scheme to minimize the sum mean squared error (SMSE) over multiple users. We further simplify the implementation of the proposed algorithm exploiting the inherent channel correlation between adjacent OFDM subcarriers. Simulations show that the proposed user assignment algorithm exhibits near optimal performance and that reducing the computational load causes minimal performance loss.
\end{abstract}

\section{INTRODUCTION}

It is now widely accepted that multiple input multiple output (MIMO) systems increase the link reliability and/or spectral efficiency of multiuser wireless communications [1]. Moreover, when channel state information (CSI) is available at the transmitter, linear precoding can be used to further improve system performance by tailoring the transmission to the instantaneous channel conditions [2] while retaining the benefits of all-linear processing. Recent work has further extended this notion to the multiuser downlink [3]-[5] wherein a single transmitter communicates simultaneously with multiple users. In this case, some knowledge of the CSI at the transmitter is mandatory for the precoding.

The literature contains various linear precoding schemes for multiuser communications. Most recently, Khachan et al. [4] consider a multiuser MIMO system with multiple data streams per user and present an algorithm that jointly optimizes the power allocation and transmit and receive filters (precoders and decoders) for all users. Given $M$ transmit antennas, the scheme can be used to communicate with up to $M$ users simultaneously. The algorithm minimizes the sum mean squared error (min SMSE) between the transmitted and received signals under a constraint of total transmitted power. The same problem is also considered in [5] where uplinkdownlink duality is used to cast the problem as a semi-definite programming convex optimization problem.

On a different front, orthogonal frequency division multiplexing (OFDM) is a simple, and now well-accepted, technique to mitigate the effects of intersymbol interference in frequency selective channels [6]. OFDM converts a broadband frequency selective channel to a series of narrowband channels by transmitting data in parallel over many subcarriers. The multiuser/multiple access version (OFDMA) assigns subcarriers to users making simultaneous communication with multiple users possible. Traditionally, in OFDMA, each subcarrier is assigned to a single user. Combining OFDM with MIMO, producing so called MIMO-OFDM, significantly reduces receiver complexity in wireless multiuser broadband systems [7], thus making it a competitive choice for future broadband wireless communication systems.

In theory, linear precoding can easily be extended to MIMOOFDM systems by applying the precoding algorithm to each subcarrier independently. This is, of course, computationally prohibitive and in [8] we present more practical and computationally efficient methods to extend linear precoding to MIMOOFDM systems by exploiting the inherent channel correlation across subcarriers. However, the work there assumes that the base station communicates with the same set of users on all subcarriers.

In systems allowing multiple users to share the same subcarrier, the number of users assigned to each subcarrier is limited by the number of transmit antennas at the base station. The total pool of users is, on the other hand, typically much larger. The base station must therefore select which users get to transmit on each given subcarrier. When the channels of the users vary independently the optimal strategy for traditional OFDMA is to select, on each subcarrier, the user with the best channel. This results in multiuser diversity, a concept first introduced in [9] and further studied in [10]. Multiuser diversity combined with spatial diversity (resulting from using multiple antennas) delivers large gains in average system throughput [11].

Due to the resulting mutual interference, this simple assignment scheme is no longer optimal when multiple users share subcarriers. In order to achieve the optimal multiuser diversity gain, we need to perform a computationally prohibitive exhaustive search over all possible combinations of users. Various suboptimal solutions have been considered in the literature to solve this problem for single carrier MIMO systems. For instance, in [12] the authors use zero-forcing beamforming and propose a suboptimal user selection scheme for MIMO broadcast channels that tries to maximize the sumrate throughput. The users are selected based on their singular values and eigenvectors to achieve maximum orthogonality. 
The literature, however, contains a limited number of studies of user selection in MIMO-OFDM systems that allow multiple users to transmit on each subcarrier. Pan et al. [13] propose a solution that uses dirty paper coding (DPC) to allow for simultaneous transmission of multiple users. User ordering associated with DPC poses a challenging problem there. Zhang and Ben Letaief [14] also consider this problem. They use zero forcing to suppress co-channel interference and propose a bit allocation scheme that can result in users sharing subcarriers.

In this paper we consider a multiuser MIMO-OFDM system that uses linear precoding and present an SMSE-based user selection algorithm. The goal is to optimally allocate $M$ users per subcarrier (the maximum allowed) from a pool of $K$ users. We also propose methods to reduce the computational load to improve the efficiency of the user selection algorithm. We do not consider the fairness problem. Instead we rely on the fact that since the users' channels are independent and identically distributed (i.i.d) and the algorithm assigns users to subcarriers based on their channel condition, in the long run the users will be assigned an equal number of subcarriers. We also do not consider some other issues of practical importance such as phase/frequency offsets between subcarriers and imperfect CSI at the transmitter.

The rest of this paper is organized as follows: Section II presents the system model and an overview of the MIMO algorithm. Section III states the user selection problem and presents our proposed algorithm. Section IV presents an algorithm simplification as well as a complexity reduction method. Finally, Section V wraps up the paper drawing some conclusions.

\section{System Model AND MIMO Algorithm}

This section presents a flat fading single carrier multiuser MIMO model and briefly summarizes the single-carrier minimum SMSE algorithm of [4] that jointly optimizes the pre/decoding matrices and the power allocation. It also presents the extension of the MIMO model to the MIMOOFDM case.

\section{A. Single-Carrier Multiuser MIMO System Model}

We consider the same system as in [4]: a single base station equipped with $M$ antennas transmitting to $K$ decentralized users. User $k$ is equipped with $N_{k}$ antennas and $N=$ $\sum_{k=1}^{K} N_{k}$. User $k$ receives $L_{k}$ data streams from the base station and $L=\sum_{k=1}^{K} L_{k}$. Thus we have $M$ transmit antennas transmitting a total of $L$ data streams to $K$ users, who have a total of $N$ receive antennas. The symbols of each user are collected in the data vector $\mathbf{x}_{k}=\left[x_{k 1}, x_{k 2}, \ldots, x_{k L_{k}}\right]^{T}$ and the overall data vector is $\mathbf{x}=\left[\mathbf{x}_{1}^{T}, \mathbf{x}_{2}^{T}, \ldots, \mathbf{x}_{K}^{T}\right]^{T}$.

User $k$ 's data streams are processed by the transmit filter $\mathbf{U}_{k} \in \mathcal{C}^{M \times L_{k}}$ before being transmitted over the $M$ antennas. These individual precoders together form the global transmitter precoder matrix $\mathbf{U}_{M \times L}=\left[\mathbf{U}_{1}, \mathbf{U}_{2}, \ldots, \mathbf{U}_{K}\right]$. Let the downlink transmit power vector for user $k$ be $\mathbf{p}_{k}=$ $\left[p_{k 1}, p_{k 2}, \ldots, p_{k L_{k}}\right]^{T}$, with $\mathbf{p}=\left[\mathbf{p}_{1}^{T}, \ldots, \mathbf{p}_{K}^{T}\right]^{T}$, and define $\mathbf{P}_{k}=\operatorname{diag}\left\{\mathbf{p}_{k}\right\}$ and $\mathbf{P}=\operatorname{diag}\{\mathbf{p}\}$. The channel between the transmitter and user $k$ is assumed flat and is represented by the $N_{k} \times M$ matrix $\mathbf{H}_{k}^{H}$. The resulting $N \times M$ channel matrix is $\mathbf{H}^{H}$, with $\mathbf{H}=\left[\mathbf{H}_{1}, \mathbf{H}_{2}, \ldots, \mathbf{H}_{K}\right]$. The transmitter is assumed to know $\mathbf{H}$.

Based on this model, user $k$ receives a length $N_{k}$ vector

$$
\mathbf{y}_{k}=\mathbf{H}_{k}^{H} \mathbf{U} \sqrt{\mathbf{P}} \mathbf{x}+\mathbf{n}_{k},
$$

where $\mathbf{n}_{k}$ represents the additive white Gaussian noise (AWGN) at the user's receive antennas with power $\sigma^{2}$; that is, $\mathbb{E}\left[\mathbf{n}_{k} \mathbf{n}_{k}^{H}\right]=\sigma^{2} \mathbf{I}_{N_{k}}$, where $\mathbb{E}[\cdot]$ represents the expectation operator. To estimate its $L_{k}$ symbols $\mathbf{x}_{k}$ in the downlink, user $k$ processes $\mathbf{y}_{k}$ with its $L_{k} \times N_{k}$ decoder matrix $\mathbf{V}_{k}^{H}$ :

$$
\hat{\mathbf{x}}_{k}^{D L}=\mathbf{V}_{k}^{H} \mathbf{H}_{k}^{H} \mathbf{U} \sqrt{\mathbf{P}} \mathbf{x}+\mathbf{V}_{k}^{H} \mathbf{n}_{k} .
$$

The global receive filter $\mathbf{V}^{H}$ is a block diagonal decoder matrix of dimension $L \times N, \mathbf{V}=\operatorname{diag}\left[\mathbf{V}_{1}, \mathbf{V}_{2}, \cdots, \mathbf{V}_{K}\right]$.

The MIMO algorithm presented in the next section exploits the duality between the uplink and downlink of the system. We construct a virtual uplink where the uplink transmit power vector for user $k$ is $\mathbf{q}_{k}=\left[q_{k 1}, q_{k 2}, \ldots, q_{k L_{k}}\right]^{T}$, with $\mathbf{q}=\left[\mathbf{q}_{1}^{T}, \ldots, \mathbf{q}_{K}^{T}\right]^{T}$. We define $\mathbf{Q}_{k}=\operatorname{diag}\left\{\mathbf{q}_{k}\right\}$ and $\mathbf{Q}=$ $\operatorname{diag}\{\mathbf{q}\}$. The transmit and receive filters for user $k$ become $\mathbf{V}_{k}$ and $\mathbf{U}_{k}^{H}$ respectively. The received vector at the base station and the estimated uplink symbol vector for user $k$ are

$$
\begin{aligned}
\mathbf{y} & =\sum_{i=1}^{K} \mathbf{H}_{i} \mathbf{V}_{i} \sqrt{\mathbf{Q}_{i}} \mathbf{x}_{i}+\mathbf{n}, \\
\hat{\mathbf{x}}_{k}^{U L} & =\sum_{i=1}^{K} \mathbf{U}_{k}^{H} \mathbf{H}_{i} \mathbf{V}_{i} \sqrt{\mathbf{Q}_{i}} \mathbf{x}_{i}+\mathbf{U}_{k}^{H} \mathbf{n} .
\end{aligned}
$$

The transmitted symbols are assumed to be independent with unit power, i.e., $\mathbb{E}\left[\mathbf{x x}^{H}\right]=\mathbf{I}_{L}$. The noise, $\mathbf{n}$, is modelled as AWGN with $\mathbb{E}\left[\mathbf{n n}^{H}\right]=\sigma^{2} \mathbf{I}_{M}$. To ensure resolvability, in the uplink and downlink, $L \leq M$ and $L_{k} \leq N_{k}, \forall k$.

\section{B. MIMO SMSE Minimization Algorithm}

Since our proposed user selection algorithm is inspired by the MIMO algorithm presented in [4], we will briefly review this algorithm to help understand SMSE based user selection. Let $\mathbf{E}_{k}^{D L}$ be the $L_{k} \times L_{k}$ error covariance matrix of user $k$ in the downlink, where

$$
\mathbf{E}_{k}^{D L}=\mathbb{E}\left[\left(\hat{\mathbf{x}}_{k}-\mathbf{x}_{k}\right)\left(\hat{\mathbf{x}}_{k}-\mathbf{x}_{k}\right)^{H}\right] .
$$

The diagonal entries of $\mathbf{E}_{k}^{D L}$ are the MSEs of the $L_{k}$ substreams of user $k$ and thus $\operatorname{SMSE}_{k}^{D L}=\operatorname{tr}\left[\mathbf{E}_{k}^{D L}\right]$, where $\operatorname{tr}[\cdot]$ is the trace operator. The SMSE minimization problem is

$$
\min _{\mathbf{p}, \mathbf{U}, \mathbf{V}} \sum_{k=1}^{K} \operatorname{tr}\left[\mathbf{E}_{k}^{D L}\right] \quad \text { subject to : }\|\mathbf{p}\|_{1} \leq P_{\max } .
$$

Using uplink-downlink duality we can solve this problem in the uplink and transfer the result to the downlink. In the uplink, the optimal minimum MSE (MMSE) receiver is

$$
\begin{gathered}
\mathbf{U}_{k}^{M M S E}=\mathbf{J}^{-1} \mathbf{H}_{k} \mathbf{V}_{k} \sqrt{\mathbf{Q}_{k}}, \\
\text { where } \quad \mathbf{J}=\mathbf{H V Q V} \mathbf{V}^{H} \mathbf{H}^{H}+\sigma^{2} \mathbf{I}_{M} .
\end{gathered}
$$


The sum MSE of the whole system is therefore

$$
\mathrm{SMSE}=\sum_{k=1}^{K} \operatorname{tr}\left[\mathbf{E}_{k}^{U L, M M S E}\right]=L-M+\sigma^{2} \operatorname{tr}\left[\mathbf{J}^{-1}\right] .
$$

The SMSE expression in (9) is a function of two variables; uplink power allocation $\mathbf{Q}$ and uplink global transmit filter $\mathbf{V}$. We first assume that $\mathbf{V}$ is fixed. Therefore, minimizing SMSE is equivalent to minimizing the trace of $\mathbf{J}^{-1}$. The resulting optimization problem is convex in $\mathbf{Q}$ [15]:

$$
\mathbf{Q}^{\text {opt }}=\arg \min _{\mathbf{Q}} \operatorname{tr}\left[\mathbf{J}^{-1}\right] \text {, subject to } \operatorname{tr}[\mathbf{Q}]=P_{\max } .
$$

The next step is to optimize $\mathbf{V}$ for a fixed power allocation $\mathbf{Q}$. The optimal $\mathbf{v}_{k j}$ for user $k$, stream $j$, which minimizes SMSE for a given power allocation when the beamforming vectors of all other streams are fixed, is the dominant generalized eigenvector of the matrix pair $\left(\mathbf{H}_{k}^{H} \mathbf{J}_{k j}^{-2} \mathbf{H}_{k}, \mathbf{I} / q_{k j}+\mathbf{H}_{k}^{H} \mathbf{J}_{k j}^{-1} \mathbf{H}_{k}\right)$, where $\mathbf{J}_{k j}=\mathbf{J}-q_{k j} \mathbf{H}_{k} \mathbf{v}_{k j} \mathbf{v}_{k j}^{H} \mathbf{H}_{k}^{H}$ [4]. Note that while each step of the iteration is optimal, it is not guaranteed that the algorithm will converge to the globally optimal solution. The single-carrier MIMO algorithm is summarized in Table I.

TABLE I

SMSE MINIMIZATION ALGORITHM

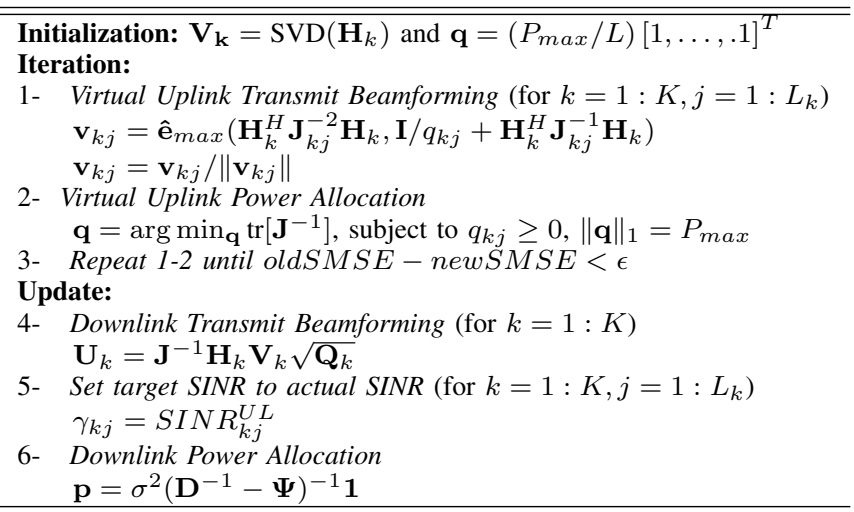

In the initialization step of the algorithm, SVD refers to singular value decomposition. The structure of the matrices $\mathrm{D}$ and $\boldsymbol{\Psi}$ and further details of the algorithm are given in [4].

\section{MIMO-OFDM System Model}

We consider a multiuser MIMO-OFDM system that employs linear precoding to communicate with $K$ users. The discrete time domain channel impulse response has $L_{t}$ taps with uniform profile and i.i.d. complex Gaussian distribution. The system has $M$ transmit antennas and uses $N_{c}$ subcarriers. The transmit filter $\mathbf{U}(n)$, corresponding to the $n$-th subcarrier, produces $M$ outputs corresponding to the $M$ antennas. However, the $N_{c}$ such outputs for each antenna are first converted to the "time domain" using an IDFT, then converted to serial form and finally augmented with a cyclic prefix. At the receiver, the $k$-th user has $N_{k}$ antennas and DFT blocks. The user attempts to decode its own $L_{k}$ streams on subcarrier $n$ by first removing the cyclic prefix, converting to parallel form, applying the
DFT, and filtering using the decoder matrix $\mathbf{V}_{k}(n)$. The result is $N_{c}$ decoded data vectors $\hat{\mathbf{x}}_{k}(n)$, each of length $L_{k}$. The goal is to minimize the SMSE over all $K$ users and $N_{c}$ subcarriers between $\hat{\mathbf{x}}_{k}(n)$ and $\mathbf{x}_{k}(n)$.

Since all users share all $N_{c}$ subcarriers, this system can be seen as $N_{c}$ parallel MIMO systems as presented in Section IIA, each with a flat fading channel resulting from the length$N_{c}$ DFT of the multi-tap frequency selective channel. Such a MIMO-OFDM system is illustrated in Fig. 1 where just one user is shown. The linear precoding algorithm of Section II-B can thus be applied to each subcarrier independently. However, more efficient implementations are discussed in [8].

\section{USER SELECTION AlgorithM}

The approach in Section II-C and [8] assumes all $K$ users share all $N_{c}$ subcarriers, requiring $K \leq M$. In a more realistic setting however, the total pool of users may be much larger than $M(K \gg M)$. The base station must then select which users to assign to each subcarrier; a good selection scheme would yield a multiuser diversity gain. To simplify the analysis, we assume that the base station assigns the maximum number of users, $M$, to each subcarrier; however, our algorithms can be used with any valid number of users. We also assume each user selected to a given subcarrier is receiving only a single data stream (i.e., $L_{k}=1 \forall k$ ). Clearly, any efficient user selection algorithm must not only consider the user's channel on the given subcarrier but also the cochannel interference.

\section{A. Problem Statement and Simple Solutions}

Using Eqn. (9) we can formulate the minimum SMSE user selection problem as

$$
\begin{aligned}
& \min _{S_{n}} \sum_{n=1}^{N_{c}} \operatorname{tr}\left[\mathbf{J}_{S_{n}}^{-1}(n)\right] \\
& \text { subject to } \operatorname{tr}\left[\mathbf{Q}_{S_{n}}(n)\right]=P_{\max }, n=1 \ldots N_{c} \\
& S_{n} \subset \mathbb{S}, \text { and }\left|S_{n}\right|=M .
\end{aligned}
$$

where $n$ is the subcarrier index, $S_{n}$ is the set of users assigned to subcarrier $n, \mathbb{S}$ is the set of all the users in the system, and $|\cdot|$ is the cardinality operator. The formulation in Eqn. (11) is explicitly over $S_{n}$, the set of users assigned to subcarrier $n$, but implicitly over the power allocations, precoders and decoders for that subcarrier, i.e., the problem of user selection is coupled with minimizing the SMSE on each subcarrier.

The optimal solution to this problem requires an exhaustive search over all possible combinations of using $M$ users out of the pool of $K$ on each subcarrier. Each step requires solving the problem in (6) by executing the iteration steps of the algorithm in Table I. This implies a huge computational load and is simply impossible for realistic values of $K$.

Another intuitive solution to this problem is to select the users with the best channel conditions to transmit on the given subcarrier. In a MIMO environment the best channels are the ones with the largest dominant singular value (SV). The approach would be to select the users with the $M$ largest 


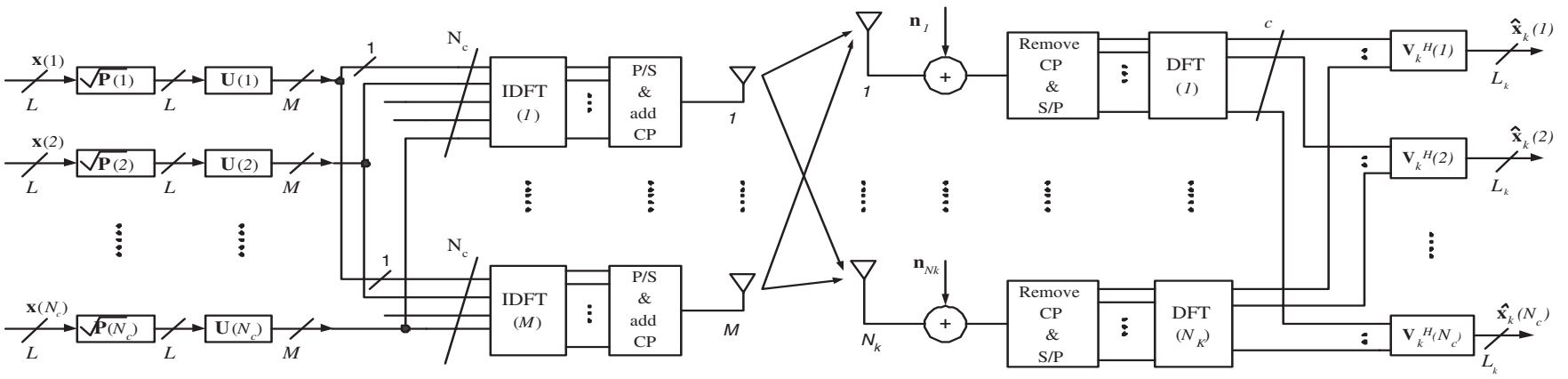

Fig. 1. MIMO-OFDM system with with $K$ users, $M$ transmit and $N_{K}$ receive antennas and $N_{c}$ subcarriers. The base station and one user are shown.

dominant SVs on each subcarrier. As we will show through simulation results, this solution yields slight performance gains over random user selection but suffers a significant loss compared to the optimal selection. This is because it fails to take into account the co-channel interference. In this paper we propose an alternative, near optimal, solution with low computational complexity. However, we begin our analysis by sketching the determination of the diversity order expected from the optimal system.

\section{B. Diversity Analysis}

For the purpose of this analysis, let us first consider a single-carrier system with a base station equipped with $M$ antennas transmitting information to $K_{c}$ single antenna users $\left(K_{c} \leq M\right)$, using MMSE precoding. At high signal-to-noise ratio (SNR), the probability density function (pdf) of the postprocessing SNR of the $k$ th user is similar to the pdf of the SNR resulting from zero forcing (ZF) [16]. Assuming that the users have unit power, the pdf of the SNR, $\rho$, is as given in [16]:

$$
p d f(\rho)=\frac{M !}{\sigma^{2}\left(K_{c}-1\right) !\left(M-K_{c}\right) !} \frac{\left(1 / \sigma^{2} \rho\right)^{K_{c}+1}}{\left(1+1 / \sigma^{2} \rho\right)^{M+1}} .
$$

Using the results of [17], the diversity order resulting from this pdf is found to be $M-K_{c}+1$, i.e., $K_{c}$ of the $M$ degrees of freedom are used to cancel interference and the remaining represent the diversity order.

Now suppose that we have a total of $K_{t}$ users and need to choose the best $K_{c}$ users for transmission. There is a total of $\mathrm{C}_{K_{t}}^{K_{t}}$ possible users combinations. Let the chosen user combination be $\left(u_{1}, u_{2}, \ldots, u_{K_{c}}\right)$, where $u_{i}$ s are the users' indices sorted in ascending order. We can pick $u_{1}$ out of $K_{t}-K_{c}+1$ possibilities $\left(u_{1} \leq K_{t}-K_{c}+1\right.$, since the user indices are in ascending order). However, once we have fixed $u_{1}$ the optimal user combination is known, thus there are no further degrees of freedom. Using order statistics [18], the cumulative distribution function (cdf) of the SNR of $u_{1}$ can be expressed as:

$$
c d f\left(\rho_{u_{1}}\right)=\operatorname{cdf}(\rho)^{K_{t}-K_{c}+1} .
$$

From (12) and (13) we deduce that the overall diversity order achieved on each subcarrier is $\left(M-K_{c}+1\right)\left(K_{t}-K+1\right)$.
This result can also be extended to the original MIMO-OFDM system described in II-C.

Having characterized the potential diversity gain incurred by user selection we next introduce the user selection algorithm.

\section{Proposed User Selection Algorithm}

The proposed user selection algorithm is inspired by the MIMO SMSE minimization algorithm. It first finds the optimal power allocation for the hypothetical case where all $K$ users are allowed to transmit on the given subcarrier. This is achieved by solving the problem in (6). Once the optimal power allocation $\mathbf{q}(n)$ is found, we choose the users corresponding to its $M$ largest entries. The algorithm is illustrated in Table II.

TABLE II

SMSE USER SELECTION ALGORITHM

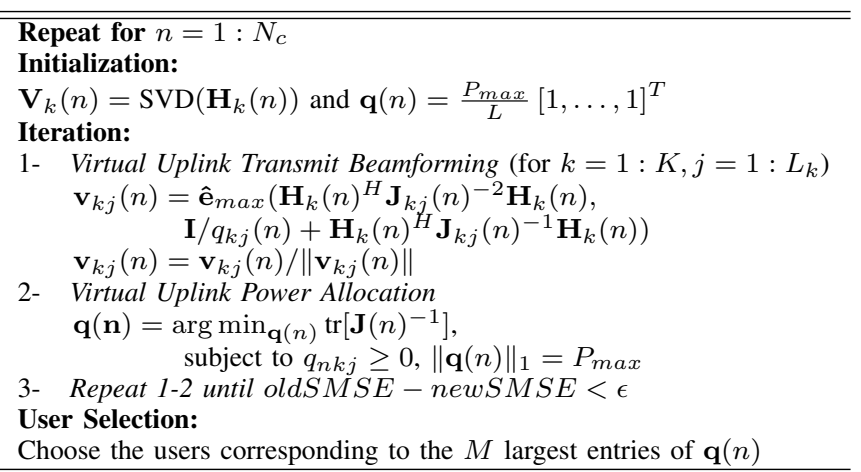

After selecting the $M$ users of a subcarrier, the algorithm in Table I is used to derive its optimal power allocation and pre/decoding matrices.

The performance of the user selection algorithm was verified and compared to other discussed solutions using Monte Carlo simulations. Figure 2 compares the performance of the proposed algorithm to the optimal, random and SV-based user selection methods. The figure illustrates the results of Monte Carlo simulations for a MIMO-OFDM system with $N_{c}=64, M=4, K=7, N_{k}=2^{1}$. The channel has $L_{t}=6$

\footnotetext{
${ }^{1}$ Note that several of the simulations uses $K=7$ because it was not possible to obtain the optimal solution for a larger value of $K$.
} 


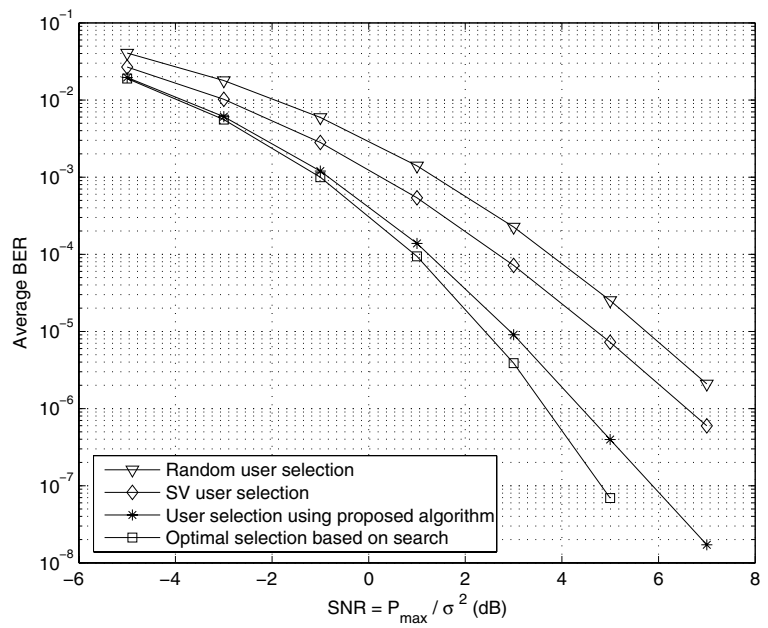

Fig. 2. Comparison of BER performance of proposed user selection algorithm to other selection methods for a MIMO-OFDM system with $M=4, K=$ $7, N_{k}=2$ and $L_{k}=1 \forall k, L_{t}=6, N_{c}=64$.

taps with uniform power profile and i.i.d. complex Gaussian distribution. The noise is assumed to be AWGN. We also assume that a cyclic prefix of appropriate length is used to avoid intersymbol interference. The plots show the average bit error rate (BER) versus average SNR per subcarrier.

From the figure we see that the SV based user selection outperforms random user selection but is significantly inferior to the optimal solution as it fails to account for the co-channel inter-user interference. Our proposed selection algorithm, on the other hand, performs close to optimal with a loss of $0.21 \mathrm{~dB}$ from optimal for a BER of $10^{-4}$.

To further quantify the performance of the proposed algorithm we examined the users sets derived by the algorithm and compared them to the corresponding optimal sets found by exhaustive search for a large number of channel realizations. We found that the two sets coincided $67 \%$ of the time. In the cases where the sets were not identical, the average discrepancy in terms of the number of users was 1.09. This further illustrates the near optimality of the proposed algorithm. The next section will examine various computational reduction methods to improve the efficiency of the algorithm.

\section{REDUCING COMPUTATION LOAD}

Although the proposed user selection algorithm discussed in the previous section has a much lower computational load than exhaustive search, it still relies on an iterative solution. Our goal in this section is to further decrease the computational load of the algorithm without significantly affecting performance.

\section{A. Minimizing Iterations}

A close inspection of the SMSE after each iteration of the presented algorithm reveals that the most significant change in SMSE happens after the first iteration. This leads us to believe that the set of users with the highest allocated power values

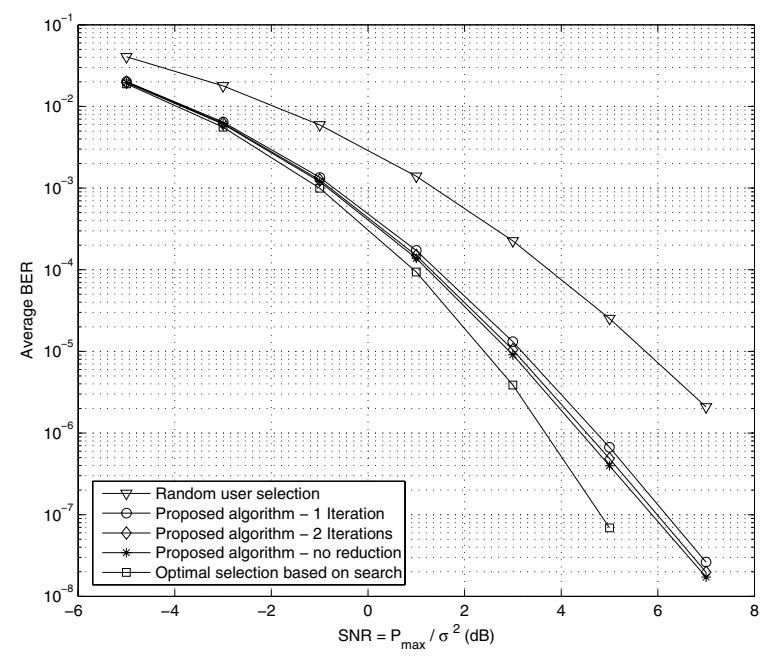

Fig. 3. Impact of iteration reduction on the proposed algorithm's BER performance for a MIMO-OFDM system with $M=4, K=7, N_{k}=2$ and $L_{k}=1 \forall k, L_{t}=6, N_{c}=64$.

does not change significantly after the first few iterations. If that is the case, then we can reduce the number of iterations by setting a limit on their total number or even removing the iteration step. We validate this hypothesis using simulations. The results for the same system settings as in the previous section are illustrated in Fig. 3.

It is clear from Fig. 3 that reducing the number of iteration to two iterations or even removing the iteration step has very little impact on the BER performance of the the proposed user selection algorithm. We conclude that the single iteration algorithm achieves the best tradeoff between performance and computational reduction.

\section{B. Clustering}

Clustering has been used to reduce the computational complexity of deriving the transmit and receive filters in MIMOOFDM systems [8], [19]. We extend this concept to further reduce the computational load of user selection algorithms.

Clustering is a simple computational reduction scheme based on the fact that adjacent OFDM subcarriers are correlated. We can therefore group adjacent subcarriers into clusters of size $L_{c l}$ and derive a single user set for each cluster based on its center subcarrier. The remaining subcarriers in the cluster would use the same user set derived for the center subcarrier. Clustering is therefore equivalent to a piecewise constant interpolation. The performance of this scheme is evaluated in Fig. 4. Given $N_{c}$ subcarriers and $L_{t}$ channel taps, we expect $L_{c l} \simeq N_{c} / L_{t}$.

We can see from Fig. 4 that clustering slightly worsens the performance of the user allocation while achieving computational gains proportional to the cluster size. A cluster size of $L_{c l}=4$ results in a penalty of less than $0.5 \mathrm{~dB}$ at a BER of $10^{-5}$, while $L_{c l}=8\left(\simeq N_{c} / L_{t}=64 / 6\right)$ results in a penalty of about $1 \mathrm{~dB}$. 


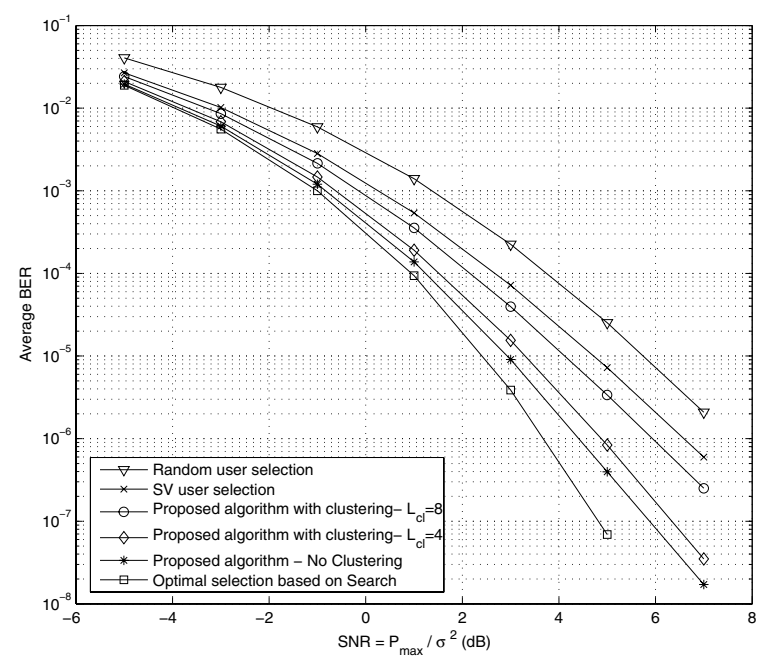

Fig. 4. Impact of clustering on the proposed algorithm's BER performance for a MIMO-OFDM system with $M=4, K=7, N_{k}=2$ and $L_{k}=$ $1 \forall k, L_{t}=6, N_{c}=64$.

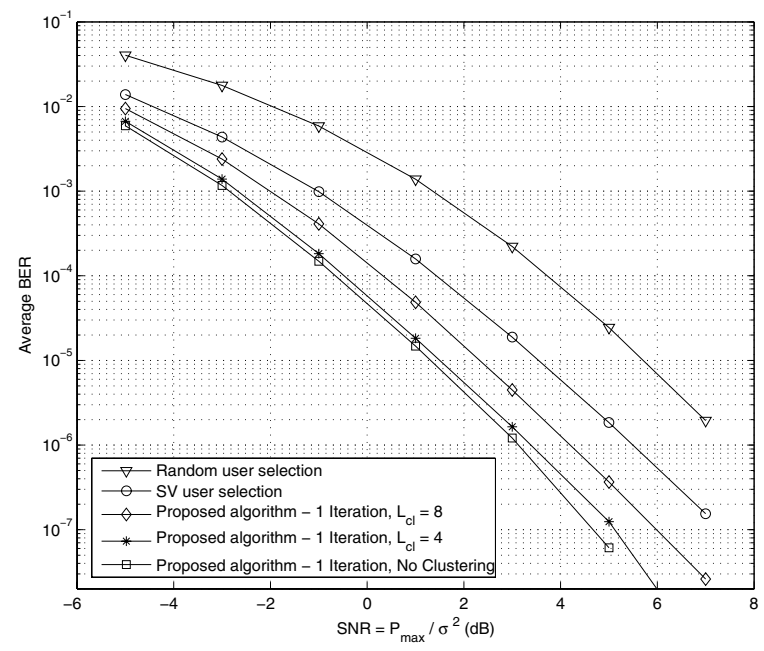

Fig. 5. Performance of the proposed algorithm and computational reduction methods for a MIMO-OFDM system with $M=4, K=40, N_{k}=2$ and $L_{k}=1 \forall k, L_{t}=6, N_{c}=64$.

Equipped with these computational simplification methods, we can now verify the performance of the proposed schemes for a more realistic total number of users $K$. Fig. 5 illustrates the performance of the system for $K=40$. The remaining system settings are the same as in previous simulations.

As illustrated in the figure the proposed schemes, under realistic system parameters, achieve a substantial gain over random user allocation and SV-based user selection. It's important to note that the optimal solution using exhaustive search cannot be obtained under these settings as there is a total of $\mathrm{C}_{4}^{40}$ user combinations for each subcarrier.

\section{CONCLUSion}

The goal of this paper is to extend the benefits of multiuser linear precoding to MIMO-OFDM systems. We focus here on the issue of user selection for the downlink of MIMO-OFDM systems using linear precoding. We formulated the minimum SMSE user allocation problem, derived the diversity order, and presented a suboptimal SMSE-based user selection algorithm inspired by the SMSE minimization algorithm presented in [4]. To make user selection more practical, we proposed a simplified version of the algorithm and reduced the overall computational load using clustering for adjacent subcarriers. Simulation results confirmed that the proposed solutions perform well under realistic settings with a large number of users.

\section{REFERENCES}

[1] G. Foschini and M. Gans, "On limits of wireless communications in a fading environment when using multiple antennas," Wireless Personal Communications, vol. 6, pp. 311-335, Mar. 1998.

[2] A. Scaglione, P. Stoica, S. Barbarossa, G. B. Giannakis, and H. Sampath, "Optimal designs for space-time linear precoders and decoders," IEEE Trans. on Signal Processing, vol. 50, no. 5, pp. 1051-1064, May 2002.

[3] A. Bourdoux and N. Khaled, "Joint TX-RX optimisation for MIMOSDMA based on a null-space constraint," in Proc. of IEEE VTC, Vancouver, Canada, Sept. 2002, pp. 171-174.

[4] A. M. Khachan, A. J. Tenenbaum, and R. S. Adve, "Linear processing for the downlink in multiuser MIMO systems with mutiple data streams," in Proc. of IEEE ICC, June 2006, pp. 4113-4118.

[5] M. Schubert, S. Shi, E. A. Jorswieck, and H. Boche, "Downlink SumMSE transceiver optimization for linear multi-user MIMO systems," in Proc. 39 Asilomar Conf. on Signals, Systems and Computers, Oct. 2005, pp. 1424-1428.

[6] A. R. S. Bahai, B. R. Saltzberg, and M. Ergen, Multi-carrier digital communications theory and applications of OFDM. New York: Springer, 2004.

[7] H. Bolcskei, D. Gesbert, and A. Paulraj, "On the capacity of OFDMbased spatial multiplexing systems," IEEE Trans. on Communications, vol. 50, no. 2, pp. 225-234, Feb. 2002.

[8] H. Karaa, R. S. Adve, and A. J. Tenenbaum, "Linear precoding for multiuser MIMO-OFDM systems," in Proc. of IEEE ICC - To Appear, June 2007.

[9] R. Knopp and P. Humblet, "Information capacity and power control in single cell multiuser communications," in Proc. of IEEE Int. Computer Conf. ICC, Seattle, WA, June 1995, pp. 331-335.

[10] P. Viswanath, D. Tse, and R. Laroia, "Opportunistic beamforming using dumb antennas," IEEE Trans. Information Theory, vol. 48, no. 6, pp. 1277-1294, June 2002.

[11] E. G. Larsson, "On the combination of spatial diversity and multiuser diversity,” IEEE Communications Letters, vol. 8, no. 8, pp. 517-519, Aug. 2004.

[12] A. Bayesteh and A. Khandani, "On the user selection for MIMO broadcast channels," in Proc. of IEEE ISIT, Sept. 2005, pp. 2325-2329.

[13] C. Pan, Y. Cai, and Y. Xu, "Adaptive subcarrier and power allocation for multiuser MIMO-OFDM systems," in Proc. of IEEE ICC, May 2005, pp. 2631-2635.

[14] Y. Zhang and K. B. Letaief, "Optimizing power and resource management for multiuser MIMO/OFDM systems," in Proc. of IEEE GLOBECOM , Dec. 2003, pp. 179-183.

[15] S. Shi and M. Schubert, "MMSE transmit optimization for multi-user multi-antenna systems," in Proc. of IEEE ICASSP, Mar. 2005.

[16] X. Shao, J. Yuan, and P. Rapajic, "Multiuser diversity for MIMO broadcast and multiple access channel with linear precoder and receiver," in Proc. of IEEE PIMRC, 2004, pp. 1597-1602.

[17] Z. Wang and G. B. Giannakis, "A simple and general parameterization quantifying performance in fading channels," IEEE Trans. on Communications, vol. 51, no. 8, pp. 1389-1398, Aug. 2003.

[18] H. A. David, Order Statistics, 2nd ed. New York: Wiley, 1981.

[19] J. Choi and R. W. Heath Jr., "Interpolation based unitary precoding for spatial multiplexing for MIMO-OFDM with limited feedback," in Proc. of IEEE GLOBECOM, Vancouver, Canada, Dec. 2004, pp. 214-218. 